\title{
Randomised controlled trial of amoxycillin clavulanate in children with chronic wet cough
}

\author{
Julie Marchant, ${ }_{1,2}^{1}$ lan Brent Masters, ${ }^{1}$ Anita Champion, ${ }^{3}$ Helen Petsky, \\ Anne B Chang ${ }^{1,4}$
}

${ }^{1}$ Queensland Children's Respiratory Centre, Royal Children's Hospital, Brisbane Australia

${ }^{2}$ Queensland Children's Medical Research Institute, University of Queensland, Brisbane, Australia ${ }^{3}$ Department of Pharmacy, Royal Children's Hospital,

Brisbane, Australia

${ }^{4}$ Menzies School of Health Research, Child Health Division, Darwin, Northern Territory, Australia

Correspondence to Dr Julie Marchant, Queensland Children's Respiratory Centre, Royal Children's Hospital, Herston, Queensland 4029,

Australia;

j.marchant@uq.edu.au

Received 14 December 2011 Accepted 26 April 2012 Published Online First 24 May 2012

\begin{abstract}
Background Despite guideline recommendations, there are no published randomised controlled trial data on the efficacy of antibiotics for chronic wet cough in children. The majority of children with chronic wet cough have protracted bacterial bronchitis (PBB), a recognised condition in multiple national guidelines. The authors conducted a parallel 1:1 placebo randomised controlled trial to test the hypothesis that a 2-week course of amoxycillin clavulanate is efficacious in the treatment of children with chronic wet cough.

Methods 50 children (median age 1.9 years, IOR 0.9-5.1) with chronic (>3 weeks) wet cough were randomised to 2 weeks of twice daily oral amoxycillin clavulanate $(22.5 \mathrm{mg} / \mathrm{kg} /$ dose $)$ or placebo. The primary outcome was 'cough resolution' defined as a $>75 \%$ reduction in the validated verbal category descriptive cough score within 14 days of treatment compared with baseline scores, or cessation of cough for $>3$ days. In selected children, flexible bronchoscopy and bronchoalveolar lavage (BAL) were undertaken at baseline.
\end{abstract}

Results Cough resolution rates (48\%) were significantly higher in children who received amoxycillin clavulanate compared with those who received placebo $(16 \%)$, $p=0.016$. The observed difference between proportions was 0.32 (95\% Cl 0.08 to 0.56). Post treatment, median verbal category descriptive score in the amoxycillin clavulanate group of 0.5 (IOR $0.0-2.0$ ) was significantly lower than in the placebo group, 2.25 (IOR 1.15-2.9) $(p=0.02)$. Pre-treatment BAL data were consistent with PBB in the majority of children, with no significant difference between groups.

Conclusion A 2-week course of amoxycillin clavulanate will achieve cough resolution in a significant number of children with chronic wet cough. BAL data support the diagnosis of PBB in the majority of these children. Clinical trial number ACTRN 12605000533695.

\section{INTRODUCTION}

Chronic cough, a common symptom presenting to general practitioners and paediatricians alike, causes a substantial amount of burden. ${ }^{1}$ Protracted bacterial bronchitis $(\mathrm{PBB})$ has recently been recognised as a common cause of chronic cough in children. ${ }^{2} 3 \mathrm{PBB}$ is defined as the presence of isolated chronic wet cough, resolution of cough with appropriate antibiotics and absence of an alternative cause for specific cough. ${ }^{4}$ We have shown previously that children with $\mathrm{PBB}$ have airway neutrophilia and an associated endobronchial infection. ${ }^{2}$ The importance of $\mathrm{PBB}$ has

\section{Key messages}

What is the key question?

- Is amoxycillin clavulanate effective in the treatment of children with chronic wet cough?

What is the bottom line?

- Compared with placebo, children with chronic wet cough who received a 2-week course of amoxycillin clavulanate were significantly more likely to achieve cough resolution at the end of 2 weeks.

\section{Why read on?}

- This study provides the first high-level evidence for the inclusion of antibiotics in paediatric cough-specific guidelines as treatment for protracted bacterial bronchitis, a common cause of chronic cough in children.

been further highlighted by speculation that $\mathrm{PBB}$ may be a precursor to chronic suppurative lung disease if left untreated. ${ }^{6}$

Since it was first described, $\mathrm{PBB}$ has been incorporated into paediatric cough guidelines in America, Britain and Australia. ${ }^{5} 78$ Current guidelines recommend antibiotic therapy for children with PBB. Presently this therapeutic advice is based on a Cochrane review, ${ }^{9}$ prospective ${ }^{2}$ and retrospective $^{4}$ observational studies. Our Cochrane review of antibiotic treatment for moist cough in children showed, with the limited data available, that antibiotics were effective with one clinical cure for every three children treated (95\% CI 2 to 4). ${ }^{9}$ However this review was based on two small studies of children with cough of $>10$ days' duration which is not the current definition of chronic cough (>4 weeks). ${ }^{5}$ Further, neither study ${ }^{10}{ }^{11}$ used validated outcome measures of cough, as none were available then. As parental reporting of cough in children is subject to bias, placebo randomised controlled trials (RCTs) and the use of validated cough outcome measures are paramount. ${ }^{12}$

To address the need for a well designed placebocontrolled RCT that addresses chronic wet cough and antibiotic treatment, ${ }^{13}{ }^{14}$ we conducted a unicentre parallel study in Brisbane. Here we present our double-blind RCT aimed to assess the efficacy of 2 weeks of oral amoxycillin clavulanate (compared with placebo) in achieving cough resolution in children with chronic wet cough. 


\section{METHODOLOGY}

Design

A parallel double-blind RCT of children with chronic wet cough.

\section{Subjects}

Entry criteria

Children (aged 6 months to $<18$ years) newly referred to the respiratory practice at the Royal Children's Hospital, Brisbane (between January 2004 and December 2006) for chronic (>3 weeks) cough and with doctor-observed moist/wet cough. ${ }^{15} 16$

\section{Exclusion criteria}

Presence of gross neurodevelopmental delay (because of increased risk of aspiration), cystic fibrosis, ex-premature $(<37$ weeks gestation) infants, chronic disease including interstitial lung disease or cardiac abnormalities, suspicion of bronchiectasis clinically, haemoptysis, antibiotic therapy received in the preceding 2 weeks, penicillin allergy or acutely unwell with fever or pneumonia.

\section{Consent and ethics approval}

Prior to enrolment, written informed consent was obtained from all parents by a nurse or doctor but not the child's treating doctor. The study was approved by the ethics committees of the Royal Children's Hospital and the University of Queensland. The study was registered with the Australian Clinical Trials Registry (ACTR) prior to commencement (ACTRN 12605000533695).

\section{Protocol}

Upon consent, enrolled children undertook the protocol (figure 1) which included a medical evaluation, including history and examination, and instructions for completion of diary cards. Children were randomised to 14 days of oral amoxycillin clavulanate suspension ( $400 \mathrm{mg} / 5 \mathrm{ml}), 22.5 \mathrm{mg} / \mathrm{kg} /$ dose twice daily, or placebo at equal volumes. The placebo, specifically made for this study by IDT Australia (Boronia, Victoria, Australia) was identical in appearance and packaging to amoxycillin clavulanate. Bottles were labelled with the patient's name and dosage in millilitres for suspension based on weight. Parents were telephoned on days 7 and 14. Participants returned the bottles of medication to check compliance after day 14, which was done by a person who was not part of the study team.

\section{Randomisation and allocation}

The randomisation (blocks of 2 or 4) list was computer generated and stratified by age ( $<6$ years and $\geq 6$ years). Concealed allocation was used whereby the enrolled child was allocated to the next sequentially numbered prescription and the medications were dispensed by the pharmacy. The allocation list and trial medications were maintained by the hospital's pharmacy. Thus the parents and members of the study were blinded to the child's allocated group until data were analysed. The medication codes were revealed in February 2011.

\section{Data collection}

Standardised data collection sheets were used for all assessments. The parents completed a daily cough diary for 28 days post enrolment to document presence and severity of cough, and any adverse reactions to medications were documented. A validated cough diary using the verbal category descriptive (VCD) score ${ }^{17}$ was used. This cough scoring system ${ }^{17}$ was used because it has the best correlation with objective cough counts as measured by a cough meter. This involves scoring the cough as listed below for each day: $0=$ no cough, $1=$ cough for one or two

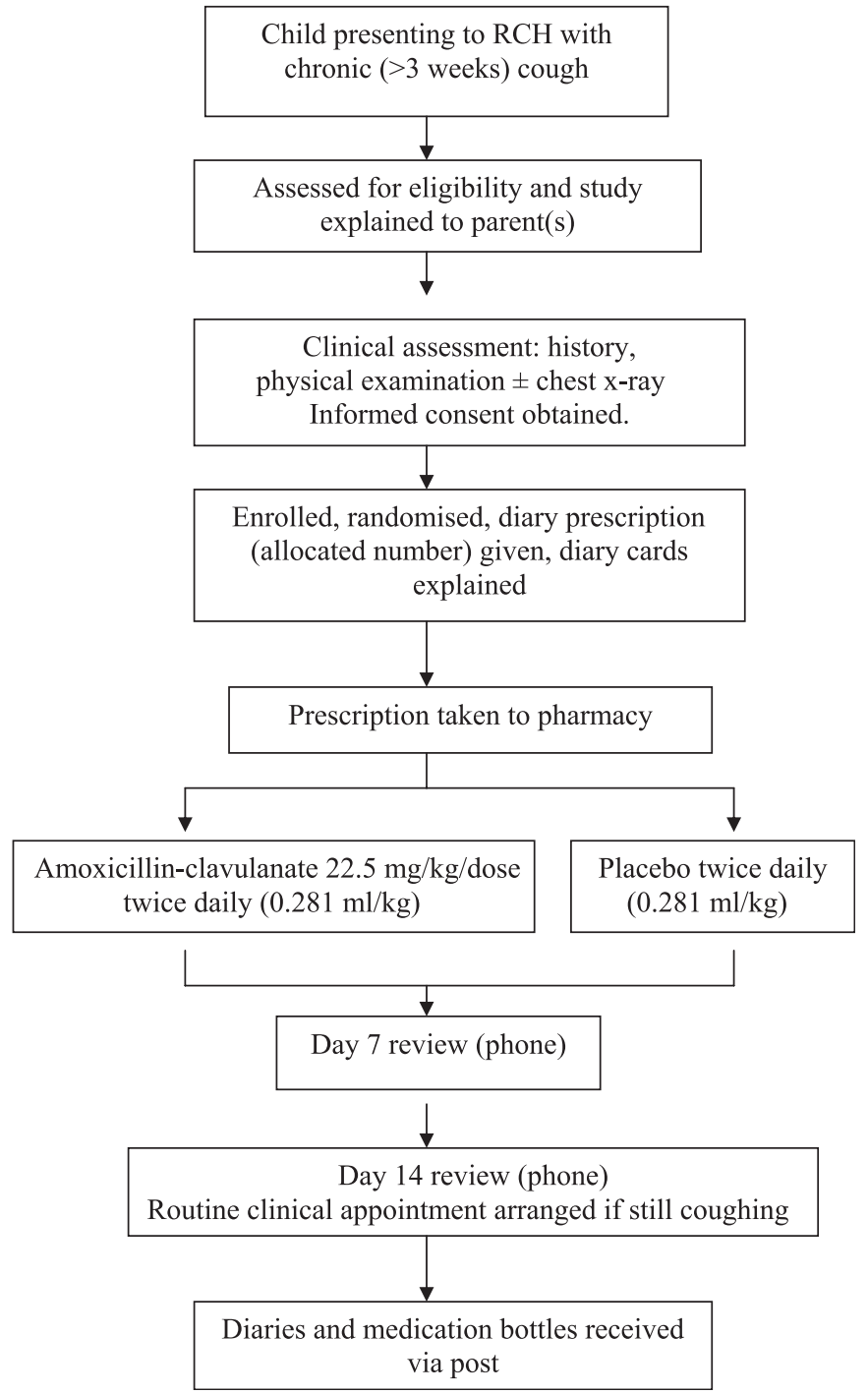

Figure 1 Protocol for children with chronic cough presenting to the Royal Children's Hospital (RCH), Brisbane.

short periods only, $2=$ cough for more then two short periods, $3=$ frequent coughing but does not interfere with school and other activities, $4=$ frequent coughing which interferes with school and other activities, $5=$ cannot perform most activities due to severe coughing.

In some children, flexible bronchoscopy was undertaken by the child's primary paediatric respiratory physician. This was not a requirement of the protocol but when planned, children received the trial medications after the bronchoscopy. When flexible bronchoscopy was undertaken, bronchoalveolar lavage (BAL) was performed as previously described, ${ }^{2}$ in accordance with the European Respiratory Society guidelines. ${ }^{18}$ The first aliquot was used for microbiological examination. The second and third aliquots were pooled for cytology and inflammatory markers. Significant bacterial growth was defined as growth of $\geq 10^{5} \mathrm{cfu} / \mathrm{ml}$ of BAL. ${ }^{19}$

\section{Outcomes}

Primary outcome was 'cough resolution', defined as an improvement in baseline cough score $(>75 \%$ reduction in cough score) at 'end of trial' or cessation of coughing for a minimum period of 3 days within the trial period. Baseline cough score was 
the average score in the 2 days immediately before trial medication commencement (days $-2,-1$ ). 'End of trial' score was the average score of the 2 days immediately following completion of 14 days of medication (days 15, 16). Secondary outcome measures were absolute change in cough score and change in VCD score over the study period.

\section{Statistical methods}

We planned for a sample size of $55-60$. At $5 \%$ significance, this provides a study power of $82 \%$ with a $20-30 \%$ dropout rate to detect a difference of $60 \%$ between groups (75\% improvement in antibiotic group, $30 \%$ in placebo group). Without dropouts, a sample size of 23 per group provided the same power for the same effect size. As the dropout rate was low, the study was ceased when 50 children were enrolled.

Data were analysed as intention to treat. Children lost to follow-up were considered failures. Data analysis was performed using SPSS V.12. Medians and IOR were used for all descriptive data. Data that were not normally distributed were analysed using non-parametric analyses: the Mann-Whitney U test was used for comparisons between the two groups. Proportions between groups were compared using $\chi^{2}$. A two-tailed $p$ value of $<0.05$ was considered significant.

\section{RESULTS}

Of 55 patients approached, 50 were enrolled and all received the treatment allocation (consort diagram, figure 2). Three children were lost to follow-up (unable to be contacted) but all 50 children were included in the final analyses for the primary outcome.

There was no significant difference between groups in any of the parameters of baseline characteristics (table 1). Similar numbers of children in both study arms had a bronchoscopy immediately prior to treatment commencement $(n=19$ in amoxycillin clavulanate group, $\mathrm{n}=18$ in placebo group). BAL data were consistent with $\mathrm{PBB}$ in the majority of children and there was no significant difference between groups for BAL total cell count, neutrophil percentage or significant bacterial growth. The BAL microbiology in the cohort identified typical respiratory pathogens including Haemophilus influenzae ( $\mathrm{n}=14,38 \%)$, Streptococcus pneumoniae ( $\mathrm{n}=9,24 \%$ ) and Moraxella catarhalis $(\mathrm{n}=7,19 \%)$. All these organisms were sensitive to amoxycillin clavulanate.

\section{Effect of intervention}

Children in the amoxycillin clavulanate group were significantly more likely to achieve cough resolution ( $n=12,48 \%)$ compared with the placebo group $(n=4,16 \%)(p=0.015)$, as summarised in table 2. The observed difference between proportions is 0.32 ( $95 \%$ CI 0.08 to 0.56$), \mathrm{OR}=4.85$, number needed to treat (NNT)

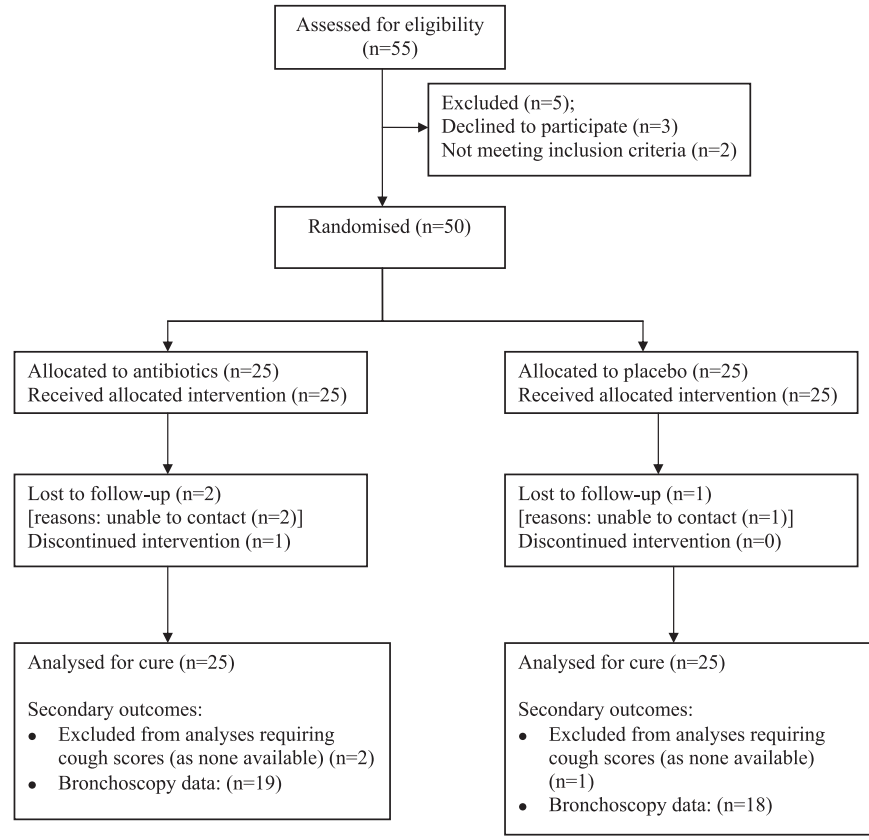

Figure 2 Consort flow diagram.

for benefit at 2 weeks was 4 (95\% CI 2 to 27). These children continued to remain cough free at study conclusion at 4 weeks and a further two children in the treatment group had ceased coughing $(n=14,56 \%)$. The data show that 10 of 13 children $(77 \%)$ in the treatment group who grew significant bacteria on BAL had ceased coughing on day 28.

While VCD cough scores were similar at baseline $(\mathrm{p}=0.549)$, end of trial VCD scores were significantly lower (ie, better) $(p=0.02)$ in the amoxycillin clavulanate group compared with the placebo group, supporting the cough resolution results (figure 3). Table 2 shows the difference between end of trial and baseline cough scores was also significantly larger in the amoxycillin clavulanate group (median change 1.5) than in the placebo group (median change 0.5) $(\mathrm{p}=0.02)$.

Side effects were seen in both groups with no significant difference between groups. Two patients in the placebo group and five in the amoxycillin clavulanate group had mild diarrhoea (difference in proportions of $-0.12,95 \% \mathrm{CI}-0.31$ to 0.07 ). One of these children (in the amoxycillin clavulanate group) also had vomiting and ceased the trial drug on day 3.

\section{DISCUSSION}

Our placebo RCT of 2 weeks of oral amoxycillin clavulanate for chronic wet cough in children showed that amoxycillin clavulanate will achieve cough resolution in a significant number of

Table 1 Subject characteristics at baseline

\begin{tabular}{|c|c|c|c|}
\hline & Treatment group $(n=25)$ & Placebo group $(n=25)$ & p Value \\
\hline Age in years, mean (SD) & $1.75(0.9,4.6)$ & $2.8(0.95,5.25)$ & 0.34 \\
\hline Men, n (\%) & $14(56)$ & $12(48)$ & 0.58 \\
\hline Cough duration in weeks, median (IOR) & $15.0(8.5-59)$ & $11.0(4.0-28)$ & 0.18 \\
\hline Smoke exposure, $\mathrm{n}(\%)$ & $8(32)$ & $7(28)$ & 0.75 \\
\hline VCD score, median (IQR) & $3.0(2.0-3.0)$ & $2.5(2.0-3.0)$ & 0.55 \\
\hline CXR abnormal, n (\%) & $9(41 \%)(n=22)$ & $6(30 \%)(n=20)$ & 0.56 \\
\hline BAL data & $\mathrm{n}=19$ & $\mathrm{n}=18$ & \\
\hline Total cell count $\left(\times 10^{9} /\right.$ litre $)$, median (IOR) & $426.0(196.0-632.0)$ & $261.0(185.5-467.5)$ & 0.45 \\
\hline$\%$ Neutrophil, median (IOR) & $38.5(13.0-58.0)$ & $34.5(8.0-66.0)$ & 0.81 \\
\hline Significant bacterial culture, n (\%) & $13(68)$ & $14(78)$ & 0.78 \\
\hline
\end{tabular}

BAL, bronchoalveolar lavage; CXR, chest $x$-ray; VCD, verbal descriptive category score. ${ }^{17}$ 
Table 2 Verbal category descriptive cough scores and cough resolution post intervention

\begin{tabular}{llcl}
\hline & $\begin{array}{l}\text { Treatment group } \\
(\mathbf{n}=\mathbf{2 5})\end{array}$ & $\begin{array}{l}\text { Placebo group } \\
(\mathbf{n}=\mathbf{2 5})\end{array}$ & p Value \\
\hline $\begin{array}{l}\text { Cough resolution, n (\%) } \\
\text { VCD score at end of }\end{array}$ & $12(48)$ & $4(16)$ & 0.015 \\
$\begin{array}{l}\text { treatment, median (IOR) } \\
\text { VCD score at end of }\end{array}$ & $0.5(0.0-2.0)$ & $2.25(1.15-2.9)$ & 0.02 \\
$\begin{array}{l}\text { study on day 28, } \\
\text { median (IOR) }\end{array}$ & $0.0(0.0-1.5)$ & $1.0 *(0.0-2.4)$ & $0.17^{*}$ \\
$\begin{array}{l}\text { Changet in VCD score, } \\
\text { median (IOR) }\end{array}$ & $1.5(0.0-2.5)$ & $0.5(-0.4-1.0)$ & 0.02 \\
\hline
\end{tabular}

*Placebo group had access to antibiotics after day 14.

†End-treatment minus baseline VCD score.

VCD, verbal descriptive category score. ${ }^{17}$

children with chronic wet cough with a NNT (for benefit at 2 weeks) of 4 . The BAL data from a subset of the children confirmed the presence of $\mathrm{PBB}$, adding weight to the study's results. Our results support major cough position statements for paediatrics in America, Britain and Australia ${ }^{5} 8$ which recommend antibiotic therapy for children with $\mathrm{PBB}$.

The American Paediatric Upper Respiratory Infection Consensus team surmised that antimicrobial treatment for prolonged cough may occasionally be indicated'. ${ }^{20}$ Despite this, there are only two randomised studies which have previously evaluated prolonged cough and antibiotic treatment. Both recruited children with $>10$ days of cough and both used shortterm antibiotic therapy of 1 week. ${ }^{10} 11$ Darelid and colleagues ${ }^{11}$ used an open randomisation design comparing erythromycin with no treatment control group. Gottfarb and Brauner ${ }^{10}$ conducted a double-blind RCT of amoxycillin clavulanate compared with placebo. A Cochrane meta-analysis of the two

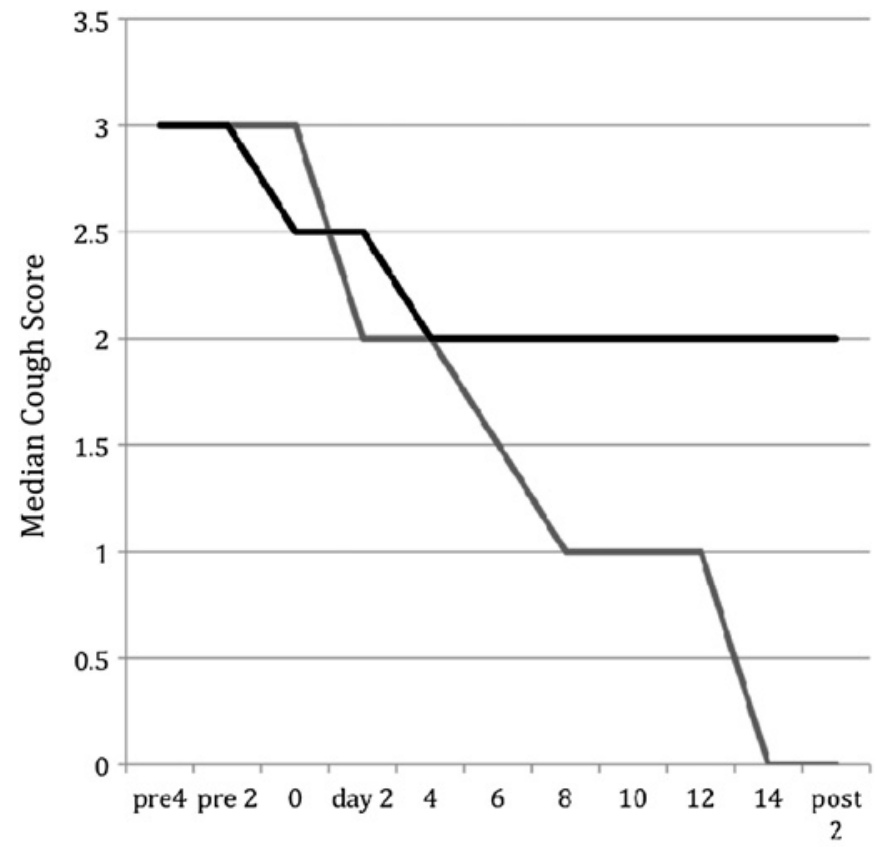

Day of RCT trial

- Treatment Grp Placebo

Figure 3 Median verbal category cough scores ${ }^{17}$ prior to commencement of study medications (pre), at baseline (day -1 ), treatment days (days 1-14), and after completion of the study (post). Dotted line = amoxycillin clavulanate group; Continuous line = placebo group. RCT, randomised controlled trial. studies found treatment with antibiotics reduced the proportion of patients not cured at follow-up (pooled OR 0.13, 95\% CI 0.06 to 0.32 ) with NNT of 3 (95\% CI 2 to 4$).{ }^{9}$ While these studies supported our previous cohort observational study that described $\mathrm{PBB},{ }^{2}$ both studies ${ }^{10}{ }^{11}$ were limited by not prospectively including cough quality, the lack of validated cough outcomes during their era, and absence of sputum or BAL as objective measures of inflammation and infection. Further, neither study had chronic ( $>4$ weeks) cough as an inclusion criteria. Our RCT addressed these shortcomings using well defined parameters of chronic cough, including only children with wet cough, validated cough outcome measures ${ }^{17}$ and using BAL measures to support clinical indices. In addition, our study has found a NNT for benefit at 2 weeks of 4 , concurring with the findings of the Cochrane meta-analysis. ${ }^{9}$

BAL data in this cohort indicate that the majority of patients had $\mathrm{PBB}$, based on a history of chronic wet cough and positive BAL fluid culture. In our previous data on $\mathrm{PBB}^{2}{ }^{3} 43 \%$ had airway neutrophilia which is similar to the percentages of $35-39 \%$ found in this study. Organisms found in this cohort were $H$ influenzae, $S$ pneumoniae and $M$ catarrhalis and are similar to previous findings ${ }^{3}$; all these organisms were sensitive to amoxycillin clavulanate. These BAL results support the diagnosis of PBB. Thus our RCT results support the current international clinical guidelines which recommend antibiotic treatment as appropriate management for this condition. 578

In our analysis, a conservative approach was used, that is, children who were treated without success were considered 'treatment failures'. The explanations for this include a different primary diagnosis, different severity of disease and presence of comorbidities. The study inclusion criteria were a chronic wet/ moist cough of $>3$ weeks in children rather than diagnoses so many diagnostic groups may be included in the cohort as there are other causes of wet cough. Thirty-seven of the 50 children were deemed severe enough (by their clinicians) to require a bronchoscopy and hence were likely a more severe group as those with 'simple' wet cough that would have responded to antibiotics were already selected out. Indeed we found it increasingly difficult to enrol children in this study as clinicians started to use more antibiotics after our study describing PBB was published. ${ }^{2}$ Thus it is not surprising that, of the patients in the treatment group who underwent a BAL, $68 \%$ had a significant bacterial culture, indicating those children without a significant growth had a different diagnosis to PBB. It is possible these 'treatment failures' had comorbidities that explained the lack of cough resolution within the study timeframe. Of the patients who did not have total clinical resolution, over half had either tracheomalacia, bronchomalacia or both and likely required a longer antibiotic course. We have previously shown that children with airway malacia have a higher severity of illness and slower rate of recovery. ${ }^{21}$ Reduced cough clearance has been implicated as a potential mechanism for increased severity in malacia conditions.

Despite the study being based in a tertiary centre, our findings are arguably applicable to primary healthcare. Participants within the study were seen in a tertiary setting and underwent bronchoscopy and other investigations. These investigations add weight to the study findings but are not necessary in the initial management of children. The necessary basic investigations, such as a chest radiograph and spirometry if older, can be undertaken in a primary care setting and we have previously shown their utility. ${ }^{22}$ When these are normal (other than peribronchiolar changes in chest radiograph) and specific cough pointers ${ }^{23}$ suggest an underlying lung disease is absent, children with chronic wet cough are likely to have endobronchial 
infection consistent with $\mathrm{PBB}$. We have previously shown this in two cross-sectional studies. ${ }^{2}{ }^{24}$ However, clinicians need to be cognisant that there are many other aetiologies of chronic wet cough. ${ }^{6}$ The occurrence of pointers to specific causes of chronic cough suggest the presence of underlying respiratory pathology, and the need for further follow-up and referral to a tertiary centre should be considered as discussed in detail previously. 522 Clinicians should also remain mindful that children with $\mathrm{PBB}$ require follow-up because $\mathrm{PBB}$ may be the first presentation of chronic suppurative lung disease. ${ }^{6}$

The limitations of our current study include patient selection and termination of the study before our planned sample size. Selection bias is a possible limitation but most children who were eligible to participate were approached and enrolled, making selection bias unlikely. Our study also lacked an objective cough outcome. However, the cough score we used had been previously validated (ie, compared with objectively measured cough counts) cross sectionally ${ }^{17}$ and in relation to change. ${ }^{25}$ Use of cough meters was not feasible as cough sensitivity cannot be validly performed in children aged $<6$ years. Our study would have been further strengthened by use of a cough-specific quality of life (QOL) measure-a validated paediatric cough-specific QOL measure became available in 2008. A further limitation of the study design is lack of follow-up over a period of months to assess the long-term value of the 2-week antibiotic therapy. Lastly, there was a long delay between completion of the study and data analyses. This was due to personal circumstances of the primary author. As the codes were only revealed after data analyses were completed, the delay would not have compromised the study's high quality. ${ }^{5}$

To our knowledge this is the first double-blind RCT to evaluate the use of antibiotics in moist/wet cough of $>3$ weeks' duration in children. The strengths of our study include use of a validated cough score as the primary outcome, concealed allocation, high follow-up rate, and availability of BAL data that confirmed the cohort of children predominantly had PBB. We conclude that amoxycillin clavulanate is effective in achieving a reduction in symptoms and cough resolution in a significant number of children with isolated chronic wet cough. This study has provided the first high-level evidence for the inclusion of antibiotics in paediatric cough-specific guidelines as treatment for $\mathrm{PBB}$ and chronic moist cough.

Acknowledgements We thank the children and parents who participated in this study. We also thank Dr Isles and Dr Francis in allowing their patients to participate in this study.

Contributors $\mathrm{JM}$ and $\mathrm{AC}$ conceived the study, participated in statistical analyses and drafted the manuscript. All authors participated in design and coordination and read and approved the final manuscript.

Funding JM was funded by the Royal Children's Hospital Foundation, Brisbane and TSANZ/Allen and Hanbury's Paediatric Respiratory Medicine Career Development Fellowship. ABC is funded by an Australian National Health and Medical Research Council Practitioner Fellowship (grant number 545216).

\section{Competing interests None.}

Ethics approval Ethics approval was provided by Royal Children's Hospital and University of Queensland Ethics Committees.
Provenance and peer review Not commissioned; externally peer reviewed.

\section{REFERENCES}

1. Marchant JM, Newcombe PA, Juniper EF, et al. What is the burden of chronic cough for families? Chest 2008:134:303-9.

2. Marchant JM, Masters IB, Taylor SM, et al. Evaluation and outcome of young children with chronic cough. Chest 2006;129:1132-41.

3. Marchant JM, Gibson PG, Grissell TV, et al. Prospective assessment of protracted bacterial bronchitis: airway inflammation and innate immune activation. Pediatr Pulmonol 2008;43:1092-9.

4. Donnelly D, Critchlow A, Everard ML. Outcomes in children treated for persistent bacterial bronchitis. Thorax 2007;62:80-4.

5. Chang $\mathbf{A B}$, Landau $\mathrm{LI}$, van Asperen PP, et al. Cough in children: definitions and clinical evaluation. Med J Aust 2006;184:398-403.

6. Chang AB, Redding GJ, Everard ML. Chronic wet cough: protracted bronchitis, chronic suppurative lung disease and bronchiectasis. Pediatr Pulmonol 2008;43:519-31.

7. Chang $\mathbf{A B}$, Glomb WB. Guidelines for evaluating cough in pediatrics: ACCP evidence-based clinical practice guidelines. Chest 2006;129(Suppl), 260S-83S

8. Shields MD, Bush A, Everard ML, et al. British Thoracic Society guidelines recommendations for the assessment and management of cough in children. Thorax 2008;63(Suppl 3);1-5.

9. Marchant JM, Morris P, Gaffney J, et al. Antibiotics for prolonged moist cough in children. Cochrane Database Syst Rev 2005;(4):CD004822.

10. Gottfarb $\mathbf{P}$, Brauner A. Children with persistent cough-outcome with treatment and role of Moraxella catarrhalis? Scand J Infect Dis 1994;26:545-51.

11. Darelid J, Lofgren S, Malmvall BE. Erythromycin treatment is beneficial for longstanding Moraxella catarrhalis associated cough in children. Scand J Infect Dis 1993:25:323-9.

12. Dales RE, White J, Bhumgara $\mathrm{C}$, et al. Parental reporting of children's coughing is biased. Eur J Epidemiol 1997:13:541-5.

13. Bailey EJ, Chang A. In children with prolonged cough, does treatment with antibiotics have a better effect on cough resolution than no treatment? Part B: clinica commentary. Paediatr Child Health 2008;13:514.

14. Bailey EJ, Chang A. In children with prolonged cough, does treatment with antibiotics have a better effect on cough resolution than no treatment? Part A: evidence-based answer and summary. Paediatr Child Health 2008;13:512-13

15. Irwin RS, Curley FJ, French CL. Chronic cough. The spectrum and frequency of causes, key components of the diagnostic evaluation, and outcome of specific therapy. Am Rev Respir Dis 1990;141:640-7.

16. Irwin RS, Boulet LP, Cloutier MM, et al. Managing cough as a defense mechanism and as a symptom. A consensus panel report of the American College of Chest Physicians. Chest 1998;114:133S-81S.

17. Chang $\mathbf{A B}$, Newman RG, Carlin J, et al. Subjective scoring of cough in children: parent-completed vs child-completed diary cards vs an objective method. Eur Respir J 1998;11:462-6.

18. de Blic J, Midulla F, Barbato A, et al. Bronchoalveolar lavage in children. ERS Task Force on Bronchoalveolar Lavage in Children. European Respiratory Society. Eur Respir J 2000;15:217-31.

19. Armstrong DS, Grimwood K, Carlin JB, et al. Lower airway inflammation in infants and young children with cystic fibrosis. Am J Respir Crit Care Med 1997:156:1197-204.

20. Dowell SF, Schwartz B, Phillips WR. Appropriate use of antibiotics for URls in children: Part II. cough, pharyngitis and the common cold. The Pediatric UR Consensus Team. Am Fam Physician 1998;58:1335-42, 1345

21. Masters IB, Zimmerman PV, Pandeya N, et al. Quantified tracheobronchomalacia disorders and their clinical profiles in children. Chest 2008;133:461-7.

22. Marchant JM, Masters IB, Taylor SM, et al. Utility of signs and symptoms of chronic cough in predicting specific cause in children. Thorax 2006;61:694-8.

23. Chang AB. Causes of cough, assessment and measurement in children. In Widdicombe JG, Chung F, Boushey H, et al, eds. Cough: Mechanisms, Causes and Therapy. Oxford: Blackwell Science, 2003.

24. Chang $\mathbf{A B}$, Faoagali J, Cox NC, et al. A bronchoscopic scoring system for airway secretions-airway cellularity and microbiological validation. Pediatr Pulmonol 2006:41:887-92.

25. Chang $\mathbf{A B}$, Phelan PD, Robertson CF, et al. Relationship between measurements of cough severity. Arch Dis Child 2003;88:57-60. 